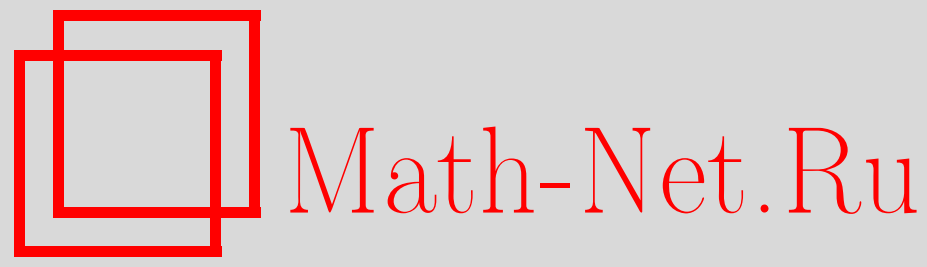

В. Д. Седых, Об эйлеровых характеристиках многообразий особенностей волновых фронтов, Функи. анализ и его прил., 2012, том 46, выпуск $1,92-96$

DOI: https://doi.org/10.4213/faa3035

Использование Общероссийского математического портала MathNet.Ru подразумевает, что вы прочитали и согласны с пользовательским соглашением

http://www.mathnet.ru/rus/agreement

Параметры загрузки:

IP : 54.197 .130 .99

26 апреля 2023 г., 09:12:56

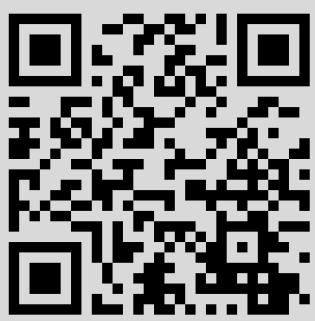


and Analytic Functions, Operator Theory Adv. Appl., vol. 202, 2010, 321-336. [7] A. Yu. Karlovich, http://arxiv.org/abs/1002.4813v1. [8] V. Kokilashvili, S. Samko, Proc. A. Razmadze Math. Inst., 131 (2003), 61-78. [9] V. Kokilashvili, S. Samko, Proc. A. Razmadze Math. Inst., 138 (2005), 106-110. [10] V. Kokilashvili, V. Paatashvili, S. Samko, in: Operator Theory Adv. Appl., vol. 170 (dedicated to 70th birthday of Prof. I. B. Simonenko), Birkhäuser, Basel, 2007, 167-186. [11] V. Kokilashvili, N. Samko, S. Samko, Math. Nachr., 280:9-10 (2007), 1145-1156. [12] В. С. Рабинович, Изв. РАН, сер. матем., 60:6 (1996), 169-200. [13] V. Rabinovich, S. Samko, Integral Equations and Operator Theory, 60:4 (2008), 507-537. [14] И. Б. Симоненко, Чинь Нгок Минь, Локалъный метод в теории одномерных сингулярных интегралъных уравнений с кусочно-непрерывными коэффиииентами. Нетеровость, Изд-во Ростовского ун-та, 1986. [15] И. Б. Симоненко, Изв. АН СССР, сер. матем., 29:3 (1965), 567-586; 29:4 (1965), 757-782. [16] I. M. Spitkovsky, J. Funct. Anal., 105:1 (1992), 129-143.

Национальный политехнический институт Мексики, e-mail: vladimir.rabinovich@gmail.com

Поступило в редакцию Университет Алгарве, Португалия e-mail: ssamko@ualg.pt

\title{
Об эйлеровых характеристиках многообразий особенностей волновых фронтов*
}

\author{
(c) 2012. В. Д. СЕдых
}

Все понятия и факты теории особенностей лежандровых отображений [1], которые мы здесь используем, кратко изложены в нашей предыдущей заметке [5].

Пусть $B$ - гладкое (класса $C^{\infty}$ ) многообразие размерности $n, E$ - гладкое $(2 n-1)$-мерное многообразие, снабженное контактной структурой, и $\rho: E \rightarrow B$ - лежандрово расслоение. Рассмотрим гладкое компактное (без края) лежандрово подмногообразие $L$ в $E$ и лежандрово отображение $f=\rho \circ i: L \rightarrow B$, где $i: L \rightarrow E-$ тождественное вложение. Образ $\mathscr{F}=f(L)$ отображения $f$ называется (волновым) фронтом. Мы будем говорить, что утверждение, касающееся лежандровых отображений или их фронтов, справедливо для отображения $f$ или фронта $\mathscr{F}$ общего положения, если оно имеет место для вложений $i$ из некоторого открытого всюду плотного подмножества в пространстве всех вложений $i: L \rightarrow E$ (в $C^{\infty}$-топологии).

При $n \leqslant 6$ особенности отображения $f$ общего положения лежандрово эквивалентны особенностям в нуле отображения

$$
\mathbb{R}^{n-1} \rightarrow \mathbb{R}^{n}, \quad(\bar{t}, \bar{q}) \mapsto\left(-S(\bar{t}, \bar{q})+\bar{t} \frac{\partial}{\partial \bar{t}} S(\bar{t}, \bar{q}),-\frac{\partial}{\partial \bar{t}} S(\bar{t}, \bar{q}), \bar{q}\right),
$$

где $\bar{t}=\left(t_{1}, \ldots, t_{k}\right), \bar{q}=\left(q_{k+1}, \ldots, q_{n-1}\right)$, а $S=S(\bar{t}, \bar{q})-$ гладкая функция одного из следующих типов ( $\mu$ целое): 


$$
\begin{array}{rll}
A_{\mu}: & S=S\left(t_{1}, \bar{q}\right)=t_{1}^{\mu+1}+q_{\mu-1} t_{1}^{\mu-1}+\cdots+q_{2} t_{1}^{2}, & 1 \leqslant \mu \leqslant n, \\
D_{\mu}^{ \pm}: & S=S\left(t_{1}, t_{2}, \bar{q}\right)=t_{1}^{2} t_{2} \pm t_{2}^{\mu-1}+q_{\mu-1} t_{2}^{\mu-2}+\cdots+q_{3} t_{2}^{2}, & 4 \leqslant \mu \leqslant n, \\
E_{6}: & S=S\left(t_{1}, t_{2}, \bar{q}\right)=t_{1}^{3}+t_{2}^{4}+q_{5} t_{1} t_{2}^{2}+q_{4} t_{1} t_{2}+q_{3} t_{2}^{2}, & \mu=6 \leqslant n
\end{array}
$$

(см. [1]). Число $\mu$ называется коразмерностью особенности. Если $\mu$ нечетное, то особенности типов $D_{\mu}^{+}$и $D_{\mu}^{-}$лежандрово эквивалентны (обозначаются через $\left.D_{\mu}\right)$. Лежандровы особенности остальных типов попарно лежандрово не эквивалентны.

Пусть $\mathbb{S}$ - свободная абелева мультипликативная полугруппа, образующими которой служат символы $A_{\mu}(\mu=1,2, \ldots), D_{2+2 k}^{-}, D_{2+2 k}^{+}, D_{3+2 k}(k=1,2, \ldots)$, $E_{6}$. Рассмотрим произвольный неединичный элемент $\mathscr{A}=X_{1} \cdots X_{p} \in \mathbb{S}$, где $X_{1}, \ldots, X_{p}$ - любой набор образующих полугруппы $\mathbb{S}$. Фронт $\mathscr{F}$ имеет особенность типа $\mathscr{A}$ в точке $y \in B$, если: 1$) f^{-1}(y)$ состоит из $p$ попарно различных точек; 2) существует порядок $x_{1}, \ldots, x_{p}$ точек из $f^{-1}(y)$, такой, что $f$ имеет в этих точках особенности типов $X_{1}, \ldots, X_{p}$ соответственно. Сумма коразмерностей особенностей типов $X_{1}, \ldots, X_{p}$ называется коразмерностью особенности типа $\mathscr{A}$ и обозначается через $\operatorname{codim} \mathscr{A}$.

Если $n \leqslant 6$, то фронт $\mathscr{F}$ общего положения имеет особенности типов $\mathscr{A} \in \mathbb{S}$, таких, что codim $\mathscr{A} \leqslant n$, во всех своих точках. Множество $\mathscr{A}_{\mathscr{F}}$ точек, в которых $\mathscr{F}$ имеет особенность типа $\mathscr{A}$, является гладким подмногообразием коразмерности $\operatorname{codim} \mathscr{A}$ в $B$. Эйлерова характеристика $\chi\left(\mathscr{A}_{\mathscr{F}}\right)$ многообразия $\mathscr{A}_{\mathscr{F}}$ (альтернированная сумма чисел Бетти групп гомологий с компактными носителями) обозначается далее через $\chi(\mathscr{A})$.

Теорема 1. Для любого компактного фронта Я્F общего положения в гладком многообразии размерности $n \leqslant 6$ справедливы следующие утверэсдения.

1) Если п нечетное, то

$$
\begin{aligned}
& \chi\left(A_{2}\right)=\chi\left(A_{3}\right)+\chi\left(A_{2} A_{1}\right)-\frac{5}{2} \chi\left(D_{5}\right)-\chi\left(D_{4}^{+} A_{1}\right)-3 \chi\left(D_{4}^{-} A_{1}\right)-2 \chi\left(A_{5}\right) \\
& \quad-2 \chi\left(A_{4} A_{1}\right)-\frac{3}{2} \chi\left(A_{3} A_{2}\right)-2 \chi\left(A_{3} A_{1}^{2}\right)-2 \chi\left(A_{2}^{2} A_{1}\right)-2 \chi\left(A_{2} A_{1}^{3}\right), \\
& \chi\left(A_{1}^{2}\right)=\frac{1}{2} \chi\left(A_{3}\right)+\chi\left(A_{2} A_{1}\right)+3 \chi\left(A_{1}^{3}\right)-\frac{7}{2} \chi\left(D_{5}\right)-\frac{1}{2} \chi\left(D_{4}^{+} A_{1}\right)-\frac{19}{2} \chi\left(D_{4}^{-} A_{1}\right) \quad-2 \chi\left(A_{5}\right)-\frac{7}{2} \chi\left(A_{4} A_{1}\right)-\frac{5}{2} \chi\left(A_{3} A_{2}\right)-\frac{11}{2} \chi\left(A_{3} A_{1}^{2}\right)-4 \chi\left(A_{2}^{2} A_{1}\right) \\
& \quad-9 \chi\left(A_{2} A_{1}^{3}\right)-20 \chi\left(A_{1}^{5}\right), \\
& \chi\left(D_{4}^{+}\right)= \frac{1}{2} \chi\left(D_{5}\right)+\chi\left(D_{4}^{+} A_{1}\right), \\
& \chi\left(D_{4}^{-}\right)= \frac{1}{2} \chi\left(D_{5}\right)+\chi\left(D_{4}^{-} A_{1}\right), \\
& \chi\left(A_{4}\right)=\chi\left(D_{5}\right)+\chi\left(A_{5}\right)+\chi\left(A_{4} A_{1}\right), \\
& \chi\left(A_{3} A_{1}\right)=\chi\left(D_{5}\right)+\chi\left(D_{4}^{+} A_{1}\right)+3 \chi\left(D_{4}^{-} A_{1}\right)+\chi\left(A_{5}\right)+\chi\left(A_{4} A_{1}\right)+\chi\left(A_{3} A_{2}\right) \quad+2 \chi\left(A_{3} A_{1}^{2}\right), \\
& \chi\left(A_{2}^{2}\right)= \frac{1}{2} \chi\left(A_{5}\right)+\chi\left(A_{3} A_{2}\right)+\chi\left(A_{2}^{2} A_{1}\right), \\
& \chi\left(A_{2} A_{1}^{2}\right)= \frac{1}{2} \chi\left(D_{5}\right)+\chi\left(A_{4} A_{1}\right)+\frac{1}{2} \chi\left(A_{3} A_{2}\right)+\chi\left(A_{3} A_{1}^{2}\right)+2 \chi\left(A_{2}^{2} A_{1}\right)+3 \chi\left(A_{2} A_{1}^{3}\right), \\
& \chi\left(A_{1}^{4}\right)=\chi\left(D_{4}^{-} A_{1}\right)+\frac{1}{2} \chi\left(A_{3} A_{1}^{2}\right)+\chi\left(A_{2} A_{1}^{3}\right)+5 \chi\left(A_{1}^{5}\right) .
\end{aligned}
$$




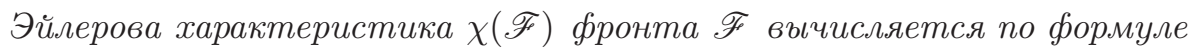

$$
\begin{aligned}
\chi(\mathscr{F})=\chi & \left(A_{1}\right)-\frac{1}{2}\left[\chi\left(A_{3}\right)+2 \chi\left(A_{2} A_{1}\right)+4 \chi\left(A_{1}^{3}\right)\right]+\frac{1}{2}\left[7 \chi\left(D_{5}\right)+\chi\left(D_{4}^{+} A_{1}\right)\right. \\
& +17 \chi\left(D_{4}^{-} A_{1}\right)+5 \chi\left(A_{5}\right)+7 \chi\left(A_{4} A_{1}\right)+5 \chi\left(A_{3} A_{2}\right) \\
& \left.+10 \chi\left(A_{3} A_{1}^{2}\right)+8 \chi\left(A_{2}^{2} A_{1}\right)+16 \chi\left(A_{2} A_{1}^{3}\right)+32 \chi\left(A_{1}^{5}\right)\right] .
\end{aligned}
$$

2) Если п четное, то

$$
\begin{aligned}
& \chi\left(A_{1}\right)=\chi\left(A_{2}\right)+2 \chi\left(A_{1}^{2}\right)-5 \chi\left(D_{4}^{-}\right)-2 \chi\left(A_{4}\right)-\frac{5}{2} \chi\left(A_{3} A_{1}\right)-2 \chi\left(A_{2}^{2}\right) \\
& -4 \chi\left(A_{2} A_{1}^{2}\right)-8 \chi\left(A_{1}^{4}\right)+13 \chi\left(E_{6}\right)+7 \chi\left(D_{6}^{+}\right)+27 \chi\left(D_{6}^{-}\right)+22 \chi\left(D_{5} A_{1}\right) \\
& +\frac{5}{2} \chi\left(D_{4}^{+} A_{2}\right)+\frac{51}{2} \chi\left(D_{4}^{-} A_{2}\right)+5 \chi\left(D_{4}^{+} A_{1}^{2}\right)+51 \chi\left(D_{4}^{-} A_{1}^{2}\right)+9 \chi\left(A_{6}\right) \\
& +14 \chi\left(A_{5} A_{1}\right)+\frac{21}{2} \chi\left(A_{4} A_{2}\right)+21 \chi\left(A_{4} A_{1}^{2}\right)+10 \chi\left(A_{3}^{2}\right)+\frac{31}{2} \chi\left(A_{3} A_{2} A_{1}\right) \\
& +31 \chi\left(A_{3} A_{1}^{3}\right)+12 \chi\left(A_{2}^{3}\right)+24 \chi\left(A_{2}^{2} A_{1}^{2}\right)+48 \chi\left(A_{2} A_{1}^{4}\right)+96 \chi\left(A_{1}^{6}\right) \text {, } \\
& \chi\left(A_{3}\right)=\chi\left(D_{4}^{+}\right)+3 \chi\left(D_{4}^{-}\right)+\chi\left(A_{4}\right)+\chi\left(A_{3} A_{1}\right)-3 \chi\left(E_{6}\right)-2 \chi\left(D_{6}^{+}\right)-7 \chi\left(D_{6}^{-}\right) \\
& -4 \chi\left(D_{5} A_{1}\right)-\chi\left(D_{4}^{+} A_{2}\right)-3 \chi\left(D_{4}^{-} A_{2}\right)-2 \chi\left(D_{4}^{+} A_{1}^{2}\right)-6 \chi\left(D_{4}^{-} A_{1}^{2}\right) \\
& -2 \chi\left(A_{6}\right)-2 \chi\left(A_{5} A_{1}\right)-\chi\left(A_{4} A_{2}\right)-2 \chi\left(A_{4} A_{1}^{2}\right)-\chi\left(A_{3}^{2}\right) \\
& \text { - } \chi\left(A_{3} A_{2} A_{1}\right)-2 \chi\left(A_{3} A_{1}^{3}\right) \text {, } \\
& \chi\left(A_{2} A_{1}\right)=\chi\left(A_{4}\right)+\chi\left(A_{3} A_{1}\right)+2 \chi\left(A_{2}^{2}\right)+2 \chi\left(A_{2} A_{1}^{2}\right)-5 \chi\left(E_{6}\right)-3 \chi\left(D_{6}^{+}\right)-7 \chi\left(D_{6}^{-}\right) \\
& -\frac{11}{2} \chi\left(D_{5} A_{1}\right)-\chi\left(D_{4}^{+} A_{2}\right)-8 \chi\left(D_{4}^{-} A_{2}\right)-2 \chi\left(D_{4}^{+} A_{1}^{2}\right)-6 \chi\left(D_{4}^{-} A_{1}^{2}\right) \\
& -4 \chi\left(A_{6}\right)-5 \chi\left(A_{5} A_{1}\right)-5 \chi\left(A_{4} A_{2}\right)-6 \chi\left(A_{4} A_{1}^{2}\right)-4 \chi\left(A_{3}^{2}\right) \\
& -\frac{11}{2} \chi\left(A_{3} A_{2} A_{1}\right)-6 \chi\left(A_{3} A_{1}^{3}\right)-6 \chi\left(A_{2}^{3}\right)-8 \chi\left(A_{2}^{2} A_{1}^{2}\right)-8 \chi\left(A_{2} A_{1}^{4}\right) \text {, } \\
& \chi\left(A_{1}^{3}\right)=\chi\left(D_{4}^{-}\right)+\frac{1}{2} \chi\left(A_{3} A_{1}\right)+\chi\left(A_{2} A_{1}^{2}\right)+4 \chi\left(A_{1}^{4}\right)-\frac{3}{2} \chi\left(E_{6}\right)-\chi\left(D_{6}^{+}\right)-5 \chi\left(D_{6}^{-}\right) \\
& -5 \chi\left(D_{5} A_{1}\right)-\frac{1}{2} \chi\left(D_{4}^{+} A_{2}\right)-\frac{11}{2} \chi\left(D_{4}^{-} A_{2}\right)-\chi\left(D_{4}^{+} A_{1}^{2}\right)-16 \chi\left(D_{4}^{-} A_{1}^{2}\right) \\
& \text { - } \chi\left(A_{6}\right)-\frac{5}{2} \chi\left(A_{5} A_{1}\right)-\frac{3}{2} \chi\left(A_{4} A_{2}\right)-5 \chi\left(A_{4} A_{1}^{2}\right)-2 \chi\left(A_{3}^{2}\right) \\
& -\frac{7}{2} \chi\left(A_{3} A_{2} A_{1}\right)-\frac{19}{2} \chi\left(A_{3} A_{1}^{3}\right)-2 \chi\left(A_{2}^{3}\right)-6 \chi\left(A_{2}^{2} A_{1}^{2}\right)-16 \chi\left(A_{2} A_{1}^{4}\right) \\
& -40 \chi\left(A_{1}^{6}\right) \text {, } \\
& \chi\left(D_{5}\right)=\chi\left(E_{6}\right)+\chi\left(D_{6}^{+}\right)+\chi\left(D_{6}^{-}\right)+\chi\left(D_{5} A_{1}\right), \\
& \chi\left(D_{4}^{+} A_{1}\right)=\chi\left(D_{6}^{+}\right)+\frac{1}{2} \chi\left(D_{5} A_{1}\right)+\chi\left(D_{4}^{+} A_{2}\right)+2 \chi\left(D_{4}^{+} A_{1}^{2}\right), \\
& \chi\left(D_{4}^{-} A_{1}\right)=\chi\left(D_{6}^{-}\right)+\frac{1}{2} \chi\left(D_{5} A_{1}\right)+\chi\left(D_{4}^{-} A_{2}\right)+2 \chi\left(D_{4}^{-} A_{1}^{2}\right), \\
& \chi\left(A_{5}\right)=\chi\left(E_{6}\right)+2 \chi\left(D_{6}^{-}\right)+\chi\left(A_{6}\right)+\chi\left(A_{5} A_{1}\right), \\
& \chi\left(A_{4} A_{1}\right)=\chi\left(E_{6}\right)+\chi\left(D_{5} A_{1}\right)+\chi\left(A_{6}\right)+\chi\left(A_{5} A_{1}\right)+\chi\left(A_{4} A_{2}\right)+2 \chi\left(A_{4} A_{1}^{2}\right) \text {, } \\
& \chi\left(A_{3} A_{2}\right)=\chi\left(D_{6}^{+}\right)+\chi\left(D_{6}^{-}\right)+\chi\left(D_{4}^{+} A_{2}\right)+3 \chi\left(D_{4}^{-} A_{2}\right) \\
& +\chi\left(A_{6}\right)+\chi\left(A_{4} A_{2}\right)+2 \chi\left(A_{3}^{2}\right)+\chi\left(A_{3} A_{2} A_{1}\right),
\end{aligned}
$$




$$
\begin{aligned}
& \chi\left(A_{3} A_{1}^{2}\right)=\chi\left(D_{6}^{-}\right)+\chi\left(D_{5} A_{1}\right)+\chi\left(D_{4}^{+} A_{1}^{2}\right)+3 \chi\left(D_{4}^{-} A_{1}^{2}\right) \\
& +\chi\left(A_{5} A_{1}\right)+\chi\left(A_{4} A_{1}^{2}\right)+\chi\left(A_{3}^{2}\right)+\chi\left(A_{3} A_{2} A_{1}\right)+3 \chi\left(A_{3} A_{1}^{3}\right), \\
& \chi\left(A_{2}^{2} A_{1}\right)=\frac{1}{2} \chi\left(E_{6}\right)+\frac{1}{2} \chi\left(A_{5} A_{1}\right)+\chi\left(A_{4} A_{2}\right)+\chi\left(A_{3} A_{2} A_{1}\right)+3 \chi\left(A_{2}^{3}\right)+2 \chi\left(A_{2}^{2} A_{1}^{2}\right), \\
& \chi\left(A_{2} A_{1}^{3}\right)=\frac{1}{2} \chi\left(D_{5} A_{1}\right)+\chi\left(D_{4}^{-} A_{2}\right) \\
& +\chi\left(A_{4} A_{1}^{2}\right)+\frac{1}{2} \chi\left(A_{3} A_{2} A_{1}\right)+\chi\left(A_{3} A_{1}^{3}\right)+2 \chi\left(A_{2}^{2} A_{1}^{2}\right)+4 \chi\left(A_{2} A_{1}^{4}\right), \\
& \chi\left(A_{1}^{5}\right)=\chi\left(D_{4}^{-} A_{1}^{2}\right)+\frac{1}{2} \chi\left(A_{3} A_{1}^{3}\right)+\chi\left(A_{2} A_{1}^{4}\right)+6 \chi\left(A_{1}^{6}\right) \text {. }
\end{aligned}
$$

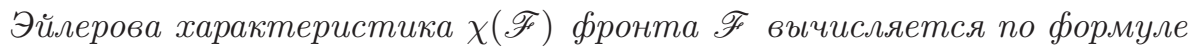

$$
\begin{aligned}
\chi(\mathscr{F})=- & \chi\left(A_{1}^{2}\right)+2 \chi\left(D_{4}^{-}\right)+\chi\left(A_{4}\right)+\chi\left(A_{3} A_{1}\right)+\chi\left(A_{2}^{2}\right)+2 \chi\left(A_{2} A_{1}^{2}\right)+5 \chi\left(A_{1}^{4}\right) \\
& -6 \chi\left(E_{6}\right)-3 \chi\left(D_{6}^{+}\right)-13 \chi\left(D_{6}^{-}\right)-11 \chi\left(D_{5} A_{1}\right)-\chi\left(D_{4}^{+} A_{2}\right)-13 \chi\left(D_{4}^{-} A_{2}\right) \\
& -2 \chi\left(D_{4}^{+} A_{1}^{2}\right)-28 \chi\left(D_{4}^{-} A_{1}^{2}\right)-4 \chi\left(A_{6}\right)-7 \chi\left(A_{5} A_{1}\right)-5 \chi\left(A_{4} A_{2}\right) \\
& -11 \chi\left(A_{4} A_{1}^{2}\right)-5 \chi\left(A_{3}^{2}\right)-8 \chi\left(A_{3} A_{2} A_{1}\right)-17 \chi\left(A_{3} A_{1}^{3}\right)-6 \chi\left(A_{2}^{3}\right) \\
& -13 \chi\left(A_{2}^{2} A_{1}^{2}\right)-28 \chi\left(A_{2} A_{1}^{4}\right)-61 \chi\left(A_{1}^{6}\right) .
\end{aligned}
$$

В случае фронтов, имеющих лишь особенности типов $A_{\mu_{1}} \ldots A_{\mu_{p}}$ (коранга 1), теорема 1 приводит к формулам, найденным в [3].

Замечание. Аналогично [4] можно показать, что любое линейное соотношение с вещественными коэффициентами между эйлеровыми характеристиками многообразий особенностей волнового фронта, справедливое для всех компактных фронтов общего положения во всех пространствах размерности $n \leqslant 6$, является вещественной линейной комбинацией соотношений из теоремы 1.

Доказательство теоремы 1 основано на следующем утверждении.

Теорема 2. Для любого компактного фронта Ғ общего положения в гладком многообразии размерности $n \leqslant 6$ и для любого неединичного элемента $\mathscr{A} \in \mathbb{S}$, такого, что codim $\mathscr{A} \leqslant n$,

$\sum_{X \in \mathbb{S}}(-1)^{\operatorname{codim} X} J_{\mathscr{A}}(X) \chi\left(X_{\mathscr{F}}\right)= \begin{cases}2(-1)^{n} \chi\left(\mathscr{A}_{\mathscr{F}}\right), & \text { если codim } \mathscr{A} \equiv n-1(\bmod 2), \\ 0, & \text { если codim } \mathscr{A} \equiv n(\bmod 2),\end{cases}$

где сумма берется по всем $X \in \mathbb{S}$, таким, что соdim $X \in[\operatorname{codim} \mathscr{A}+1, n]$, a $J_{\mathscr{A}}(X)$ - индекс примыкания особенностей типа $X \kappa$ особенностям типа $\mathscr{A}$.

Эта формула следует из аддитивности эйлеровой характеристики. Индексы $J_{\mathscr{A}}(X)$ вычислены в [5].

Следствие 3. Числа изолированных особенностей типов $D_{5}, A_{5}, A_{3} A_{2} u$ $A_{3} A_{1}^{2}$ у любого компактного фронта общего положения в 5 -мерном пространстве являются четными. Числа изолированных особенностей типов $D_{5} A_{1}$, $A_{3} A_{2} A_{1}$ и $A_{3} A_{1}^{3}$ у любого компактного фронта общего положения в 6 -мерном пространстве являются четными; числа особенностей типов $E_{6}$ и $A_{5} A_{1}$ имеют одинаковую четность. 
Условия сосуществования особенностей волновых фронтов, указанные в следствии 3, вытекают также из свойств комплекса лежандровых мультиособенностей, построенного в [2].

\section{ЛитеРАтУРА}

[1] В. И. Арнольд, Особенности каустик и волновых фронтов, Фазис, М., 1996. [2] В. А. Васильев, Лагранжевы и лежандровы характеристические классы, МЦНМО, M., 2000. [3] В. Д. Седых, Функц. анализ и его прил., 37:2 (2003), 52-64. [4] В. Д. Седых, Функц. анализ и его прил., 38:4 (2004), 73-78. [5] В. Д. Седых, Функц. анализ и его прил., 44:3 (2010), 88-91.

РГУ нефти и газа им. И. М. Губкина e-mail: vdsedykh@gmail.com

Поступило в редакцию 30 сентября 2009 г.

Заведующая редакцией и научный редактор Г. М. Цукерман

Сдано в набор 23.12.2011. Подписано к печати 27.01.2012. Формат 70×100/16 Печать офсетная. Усл. печ. л. 7,8. Усл. кр.-отт. 1,6 тыс. Бум. л. 3,0

Уч.-изд. л. 8,0. Тираж 202 экз. Заказ 2072.

Учредитель: Российская академия наук

Адрес редакции: 117966 Москва, ГСП-1, ул. Губкина 8, комн. 624. Тел. 938-37-56

Издатель: Российская академия наук, Издательство «Наука», 117997 Москва, Профсоюзная, ул. 90

Отпечатано в ППП «Типография «Наука», 121099 Москва, Шубинский пер., 6 